\title{
Obesity paradox in coronary artery bypass grafting: Myth or reality?
}

\author{
Umberto Benedetto, MD, PhD, Carla Danese, and Massimiliano Codispoti, MB ChB, MD, FRCS, FRCS C-Th
}

Objective: A high body mass index has been suggested to be protective in patients with coronary artery disease and in those undergoing coronary artery bypass grafting (CABG). However, these conflicting results might be related to the different risk profiles among the various body mass index categories. We sought to clarify the effect of varying degrees of excessive body weight on hard clinical outcomes in patients undergoing CABG.

Methods: A retrospective analysis of prospectively collected data was conducted to investigate the effect of a high body mass index on early and late mortality after first-time isolated CABG. Propensity score matching was used to adjust for confounding factors.

Results: The study sample consisted of 3269 normal weight, 6662 overweight, 3821 obese, and 211 morbidly obese patients. After matching, early mortality was not affected by overweight (mean difference, $0.7 \% ; 95 \%$ confidence interval $[\mathrm{CI}],-0.2 \%$ to $1.5 \%$ ), obesity (mean difference, $0.5 \% ; 95 \% \mathrm{CI},-0.7 \%$ to $1.7 \%$ ), and morbid obesity (mean difference, $1.6 \% ; 95 \% \mathrm{CI},-1.0 \%$ to $1.0 \%$ ), regardless of the patients' risk profile according to the European system for cardiac operative risk evaluation. Overweight status was not protective for late death (hazard ratio, $1.05 ; 95 \% \mathrm{CI}, 0.9-1.08 ; P=.4$ ). Compared with normal weight patients, both obese and morbidly obese patients had a higher risk of late death (hazard ratio, $1.22 ; 95 \% \mathrm{CI}, 1.07-2.66 ; P=.006$ for obese patients; hazard ratio, $1.36 ; 95 \% \mathrm{CI}, 0.74-2.49 ; P=.3$ for morbidly obese patients).

Conclusions: Obesity did not increase operative mortality, but it was associated with reduced late survival in patients undergoing primary isolated CABG. Our results raise concerns in supporting any protective effect of obesity in cardiovascular disease, specifically in patients undergoing surgical myocardial revascularization. (J Thorac Cardiovasc Surg 2014;147:1517-23)

According to the World Health Organization, the prevalence of obesity nearly doubled from 1980 to 2008, with more than 1.4 billion adults being overweight and more than half a billion obese. At least 2.8 million people die annually as a result of being overweight or obese. Obesity is a well known cardiometabolic risk factor, and the prevalence of almost every cardiovascular disease, including hypertension, heart failure, peripheral arterial disease, atrial fibrillation, and coronary artery disease, is markedly increased in the presence of obesity. ${ }^{1}$ However, despite these adverse effects of obesity, numerous studies have suggested the existence of an "obesity paradox," according to which normal weight patients with established coronary artery disease have a worse clinical prognosis than their more overweight or obese counterparts. ${ }^{2}$

The number of obese patients referred for coronary artery bypass grafting $(\mathrm{CABG})$ has been increasing. ${ }^{3}$ Obese patients have traditionally been considered at higher risk

\footnotetext{
From the Cardiothoracic Department, Papworth Hospital, National Health Service Foundation Trust, Papworth Everard, Cambridge, United Kingdom.

Disclosures: Authors have nothing to disclose with regard to commercial support.

Received for publication March 18, 2013; revisions received May 2, 2013; accepted for publication May 23, 2013; available ahead of print July 18, 2013.

Address for reprints: Massimiliano Codispoti, MB ChB, MD, FRCS, FRCS C-Th, Cardiothoracic Department, Papworth Hospital, National Health Service Foundation Trust, Papworth Everard, Cambridge CB23 3RE, UK (E-mail: m.codispoti@ nhs.net).

$0022-5223 / \$ 36.00$

Copyright (c) 2014 by The American Association for Thoracic Surgery

http://dx.doi.org/10.1016/j.jtcvs.2013.05.028
}

of perioperative morbidity and mortality, and, occasionally, they have not even been considered suitable for surgery solely because of their obese status. However, conflicting results have recently been reported, ${ }^{4-8}$ with some investigations showing that a high body mass index (BMI) might even have a beneficial effect on early and late mortality, supporting the existence of an "obesity paradox" in patients undergoing CABG. ${ }^{4-7}$ The biological basis for this seemingly contradictory clinical observation remains unclear. One possible explanation can be found in the different characteristics of patients in the obese and normal weight groups. For example, overweight and obese patients were reported to be significantly younger than the normal weight patients at the time of CABG. ${ }^{4-7}$ Therefore, the different risk profile, rather than obesity per se, might have accounted for the apparently better prognosis of this patient population. To date, only a few studies have reported on the effect of a high BMI on survival after CABG, with several limitations, including a small sample size and significant differences in baseline characteristics in patients across the different BMI categories. Because obesity is a potentially treatable condition, ${ }^{9}$ additional studies are warranted to confirm or refute this "obesity paradox" and to improve the treatment of such challenging patients.

Using a large database with 17 years of follow-up, we investigated the effect of a high BMI on the early and late mortality of patients undergoing first-time isolated CABG. 


$$
\begin{aligned}
& \text { Abbreviations and Acronyms } \\
& \text { BMI = body mass index } \\
& \mathrm{CABG}=\text { coronary artery bypass grafting } \\
& \mathrm{CI}=\text { confidence interval } \\
& \text { EuroSCORE }=\text { European system for cardiac } \\
& \text { operative risk evaluation } \\
& \text { HR = hazard ratio } \\
& \text { OR } \quad=\text { odds ratio }
\end{aligned}
$$

\section{METHODS \\ Study Population}

The present study was performed with permission from the local medical ethics committee. We retrospectively analyzed prospectively collected data from the surgical database of Papworth Hospital (Cambridge, UK). This database is maintained by a team of full-time clinical information analysts, who are responsible for continuous prospective data collection as a part of a continuous audit process that is linked to a national database. Data collection is validated regularly. Information about death from any cause is obtained from the General Register Office approximately 1 week after the event. Patients undergoing first-time isolated CABG from March 1996 to May 2012 were included in the present analysis. Patients with missing data on important baseline clinical covariates were excluded from the study sample.

The patients were divided into 5 groups according to their BMI: underweight (BMI $<18.5 \mathrm{~kg} / \mathrm{m}^{2}$ ), normal weight (BMI, 18.5-24.9 kg/m ${ }^{2}$ ), overweight (BMI, $25-29.9 \mathrm{~kg} / \mathrm{m}^{2}$ ), obese (BMI, 30-34.9 kg/m ${ }^{2}$ ), and morbidly obese $\left(B M I \geq 35 \mathrm{~kg} / \mathrm{m}^{2}\right)$. Because of the small sample size $(\mathrm{n}=67)$, underweight patients were not included in the present analysis.

The primary endpoints were all-cause early (within 30 days or hospital discharge, whichever was earlier) and late ( $>30$ days or after hospital discharge) death. We focused on all-cause death, which represents the most robust and unbiased index event, because no adjudication was required, thus avoiding inaccurate or biased documentation and clinical assessments.

\section{Statistical Analysis}

Baseline risk factors were selected among the variables included in the European system for cardiac operative risk evaluation (EuroSCORE). ${ }^{10}$ The year of the operation was included in the analysis to correct for variable standards of perioperative care over time. Operative risk was derived by calculating the logistic EuroSCORE. The additive EuroSCORE was used to classify the patient risk profile into 3 groups: low risk (0-2), with an expected mortality of $<2 \%$; medium risk (3-5), with an expected mortality of $<5 \%$; and high risk $(\geq 6)$, with an expected mortality of $>6 \% .{ }^{10}$

The results are presented as the mean \pm standard deviation for continuous variables and as proportions for categorical variables, according to the stated BMI categories.

Before matching, an analysis of the unmatched patient population was conducted to assess the effect of a high BMI on early and late mortality using logistic and Cox regression analysis, respectively, after adjusting for all covariates significantly associated with the chosen endpoints on univariate analysis $(P<.5)$. The BMI was forced into the models as a categorized and continuous variable, using normal weight as the control.

Propensity score matching was used to minimize the bias related to the baseline differences among the BMI groups. ${ }^{11}$ To estimate the propensity score, 3 logistic regression models were used, in which the BMI categories were regressed on the baseline characteristics listed in Table 1. Pairs of normal weight versus overweight, obese, and morbidly obese patients were derived using 1:1 matching. To optimize the matching process, we used "optimal" matching implemented by the MatchIt package (R project, $\mathrm{R}$ Foundation for Statistical Analysis, Vienna, Austria), ${ }^{11}$ which finds the matched samples with the smallest average absolute distance across all the matched pairs. The commonly used nearest-neighbor matching method is greedy matching, in which the closest control match for each treated unit is chosen 1 at a time, without trying to minimize a global distance measure. The standardized mean difference was used to quantify the differences in the mean or prevalence between overweight and obese versus normal weight patients in the overall sample size and in the matched samples; a value $>0.10$ represented a meaningful imbalance in a given covariate. ${ }^{12}$

The difference in the probability of 30-day mortality among the BMI groups was assessed by directly estimating the difference in proportions among the BMI categories in the matched samples. ${ }^{11}$ To investigate the effect of patient risk profile on early mortality in the BMI categories, we conducted a separate analysis according to 3 EuroSCORE risk groups (low, medium, and high risk, as previously specified).

The effect of the BMI categories on the interval to late death ( $>30$ days) was assessed using Kaplan-Meier survival curves in the matched samples and the stratified log-rank method. ${ }^{12}$

All statistical analyses were performed using R, version 2.15.2 (R Foundation for Statistical Analysis).

\section{RESULTS \\ Baseline}

The study sample consisted of 3269 normal weight, 6662 overweight, 3821 obese, and 211 morbidly obese patients. The baseline characteristics of the patients according to BMI status are listed in Table 1 . Several variables had standardized differences that exceeded 0.10, indicating significant differences in the baseline characteristics among the overweight, obese, and morbidly obese patients versus the normal weight patients. Normal weight patients tended to be older, more likely to have extracardiac arteriopathy, and to have an increased serum creatinine. They were more likely to undergo nonelective CABG. Subjects with a normal BMI presented with an increased overall risk profile, as assessed by the logistic EuroSCORE $(5.9 \% \pm$ $8.5 \%)$ compared with overweight $(4.7 \% \pm 6.8 \%$; analysis of variance, $P<.05$ vs normal weight), obese $(4.3 \% \pm$ $6.9 \%, P<.05$ vs normal weight), and morbidly obese $(3.6 \% \pm 5.3 \% ; P<.05$ vs normal weight) patients.

\section{Unmatched Sample Analysis}

In the unmatched sample, crude mortality within 30 days for normal weight, overweight, obese, and morbidly obese patients was $3.81 \%, 1.99 \%, 2.23 \%$, and $1.43 \%$, respectively. After adjusting for other risk factors, overweight status showed a protective effect on early mortality compared with normal weight (odds ratio [OR], 0.62; 95\% CI, 0.48$0.81 ; P=.0005)$. However, this protective effect was no longer significant in the obese (OR, $0.79 ; 95 \% \mathrm{CI}, 0.58$ $1.08 ; P=.14)$ and morbidly obese $(\mathrm{OR}, 0.74 ; 95 \% \mathrm{CI}$, $0.22-2.41 ; P=.6)$ patients. When the analysis was conducted according to the patient risk profile, being overweight was protective for high-risk $(P=.004)$ and medium-risk $(P=.02)$ but not for low-risk $(P=.7)$ profile patients. As a continuous variable, BMI showed a U-shaped effect on the adjusted risk of early mortality with a significant risk reduction for BMI values of 26 to $32 \mathrm{~kg} / \mathrm{m}^{2}$ (Figure 1, top). 
TABLE 1. Baseline characteristics among all unmatched cases

\begin{tabular}{|c|c|c|c|c|c|c|c|}
\hline \multirow[b]{2}{*}{ Characteristic } & \multirow[b]{2}{*}{$\begin{array}{l}\text { Normal weight } \\
\quad(n=3269)\end{array}$} & \multirow[b]{2}{*}{$\begin{array}{c}\text { Overweight } \\
(\mathrm{n}=6662)\end{array}$} & \multirow[b]{2}{*}{$\begin{array}{c}\text { Obese } \\
(\mathbf{n}=\mathbf{3 8 2 1}) \\
\end{array}$} & \multirow[b]{2}{*}{$\begin{array}{c}\text { Morbidly obese } \\
(\mathbf{n}=\mathbf{2 1 1})\end{array}$} & \multicolumn{3}{|c|}{ SMD } \\
\hline & & & & & $\begin{array}{l}\text { Normal vs } \\
\text { overweight }\end{array}$ & $\begin{array}{c}\text { Normal vs } \\
\text { obese }\end{array}$ & $\begin{array}{c}\text { Normal vs } \\
\text { morbidly obese }\end{array}$ \\
\hline Age (y) & $68 \pm 9$ & $66 \pm 9$ & $64 \pm 9$ & $60 \pm 9$ & 0.17 & 0.39 & 0.83 \\
\hline Female gender $(\%)$ & 0.22 & 0.15 & 0.20 & 0.37 & 0.15 & 0.04 & 0.34 \\
\hline Creatinine $(\mathrm{mmol} / \mathrm{L})$ & $108 \pm 61$ & $108 \pm 21$ & $104 \pm 44$ & $100 \pm 33$ & 0.001 & 0.01 & 0.23 \\
\hline LV dysfunction $(\%)$ & 0.46 & 0.46 & 0.47 & 0.53 & 0.004 & 0.02 & 0.12 \\
\hline $\operatorname{IDDM}(\%)$ & 0.12 & 0.14 & 0.19 & 0.22 & 0.01 & 0.07 & 0.1 \\
\hline COPD $(\%)$ & 0.10 & 0.09 & 0.11 & 0.13 & 0.04 & 0.01 & 0.03 \\
\hline Extracardiac arteriopathy $(\%)$ & 0.10 & 0.10 & 0.09 & 0.07 & 0.01 & 0.03 & 0.1 \\
\hline CVA $(\%)$ & 0.01 & 0.01 & 0.01 & 0.01 & 0.007 & 0.01 & 0.02 \\
\hline Nonelective surgery $(\%)$ & 0.27 & 0.20 & 0.19 & 0.22 & 0.16 & 0.18 & 0.09 \\
\hline Redo surgery $(\%)$ & 0.03 & 0.03 & 0.03 & 0.04 & 0.0049 & 0.005 & 0.07 \\
\hline Year of operation (median) & 2003 & 2003 & 2004 & 2005 & 0.04 & 0.21 & 0.53 \\
\hline
\end{tabular}

Data are presented as mean \pm standard deviation, unless otherwise noted. $S M D$, Standardized mean difference; $L V$, left ventricle; $I D D M$, insulin-dependent diabetes mellitus; $C V A$, cerebrovascular accident; $C O P D$, chronic obstructive pulmonary disease.

Among the operative survivors, the mean follow-up time was $7.6 \pm 4.5$ years. Late survival at 5 years was $90.1 \% \pm$ $0.5 \%, 92.6 \% \pm 0.3 \%, 92.2 \% \pm 0.4 \%$, and $91.6 \% \pm 2.2 \%$ for normal weight, overweight, obese, and morbidly obese patients, respectively. After adjusting for other risk factors, overweight status showed a protective effect on late mortality compared with normal weight (hazard ratio [HR], 0.88; 95\% CI, 0.80-0.97; $P=.018$ ). Obesity (HR, $1.04 ; 95 \%$ CI, $0.93-1.17 ; P=.43)$ and morbid obesity (OR, $1.29 ; 95 \% \mathrm{CI}$, $0.85-1.96 ; P=.2$ ) did not influence late survival compared with normal weight.

When BMI was investigated as a continuous variable, it was shown to be significantly protective for late mortality only for a BMI of 27 to $29 \mathrm{~kg} / \mathrm{m}^{2}$ (Figure 1 , bottom).

\section{Propensity Score Matching Analysis}

After propensity matching, 3072 matched pairs of overweight versus normal weight patients were identified. The baseline characteristics of the 2 matched samples are listed in Table 2 . The standardized difference was $<0.1$ for all covariates investigated, indicating that the 2 groups were very similar. No difference was found in 30-day mortality among overweight and normal weight patients (mean difference, $0.7 \% ; 95 \% \mathrm{CI},-0.2 \%$ to $1.5 \%)$. This result was confirmed in the low-risk $(P=.4)$, medium-risk $(P=.2)$, and high-risk $(P=.1)$ patients. After a mean follow-up of $7.5 \pm 4.6$ years (range $0-17$ ), overweight patients had late survival comparable to that in the normal weight group (HR, 1.05; 95\% CI, 0.9-1.08; $P=.4$; Figure 2).

A total of 1609 matched pairs of obese versus normal weight subjects were formed, and the 2 groups were comparable in respect to all risk factors thought to influence the chosen outcome measures (Table 3). No difference was found in 30-day mortality between the obese and normal weight patients (mean difference, $0.5 \% ; 95 \% \mathrm{CI},-0.7 \%$ to $1.7 \%$ ). This result was confirmed in the low-risk
$(P=.1)$, medium-risk $(P=.1)$, and high-risk $(P=.9)$ patients. However, after a mean follow-up of $7.1 \pm 4.5$ years (range, 0-17), obesity was associated with an increased risk of late death compared with normal weight (HR, 1.22; 95\%
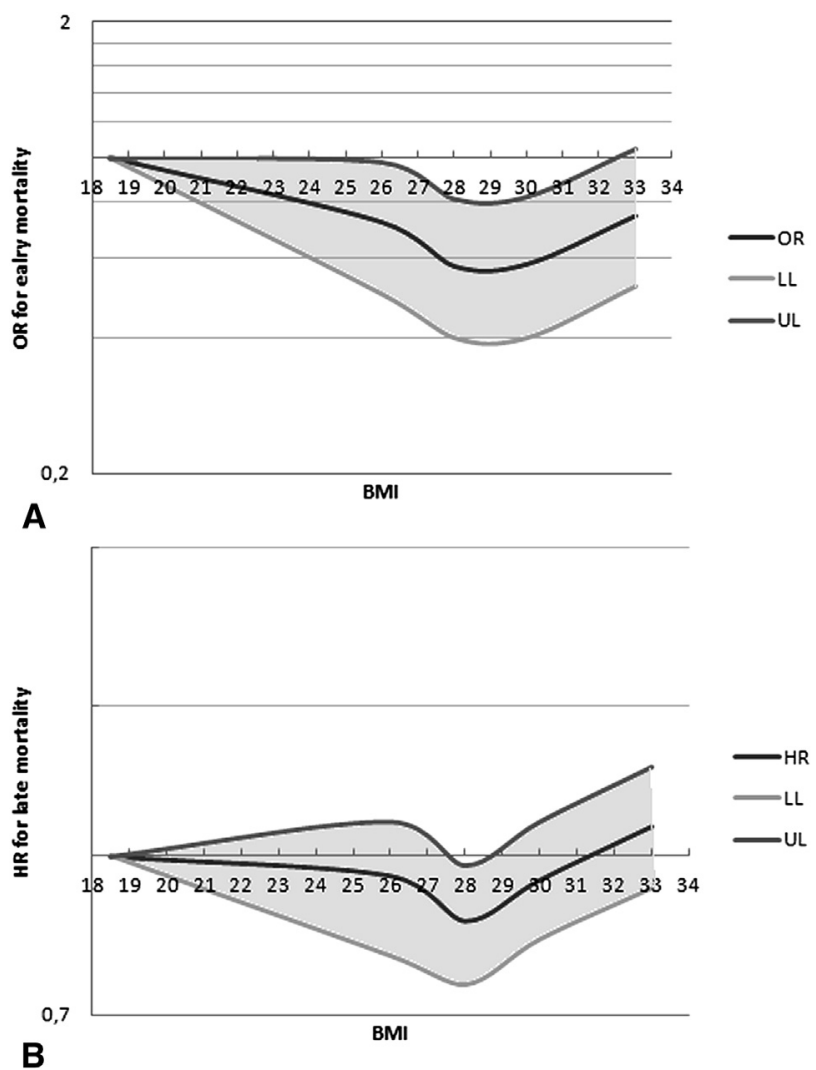

FIGURE 1. Relationship between body mass index $(B M I)$ as a continuous variable and risk for early (top) and late (bottom) mortality in the unmatched sample. $O R$, Odds ratio; $H R$, hazard ratio; $L L$, lower limit of $95 \%$ confidence interval; $U L$, upper limit of $95 \%$ confidence interval. 
TABLE 2. Baseline characteristics among normal weight and overweight subjects after matching

\begin{tabular}{lcccc}
\hline & & & \multicolumn{2}{c}{ SMD } \\
\cline { 3 - 5 } \multicolumn{1}{c}{ Variable } & Normal weight $(\mathbf{n}=\mathbf{3 0 7 2})$ & Overweight $(\mathbf{n}=\mathbf{3 0 7 2})$ & Normal vs overweight & Balance improvement (\%) \\
\hline Age (y) & 68.1 & 68.6 & 0.04 & 74 \\
Female gender (\%) & 0.21 & 0.21 & 0.007 & 95 \\
Creatinine (mmol/L) & 108 & 108 & 0.001 & 8 \\
LV dysfunction (\%) & 0.46 & 0.46 & 0.003 & 8 \\
IDDM (\%) & 0.12 & 0.12 & 0.001 & 8 \\
COPD (\%) & 0.10 & 0.10 & 0.01 & 67 \\
Extracardiac arteriopathy (\%) & 0.10 & 0.10 & 0.002 & 87 \\
CVA (\%) & 0.01 & 0.01 & 0.003 & 60 \\
Nonelective surgery (\%) & 0.26 & 0.26 & 0.002 & 98 \\
Redo surgery (\%) & 0.033 & 0.038 & 0.02 & 47 \\
Year of operation (median) & 2003 & 2003 & 0.0003 & 99 \\
\hline Danyyyyy
\end{tabular}

Data are presented as mean values, unless otherwise noted. $S M D$, Standardized mean difference; $L V$, left ventricle; IDDM, insulin-dependent diabetes mellitus; $C V A$, cerebrovascular accident; $C O P D$, chronic obstructive pulmonary disease.

CI, 1.07-2.66; $P=.006$; Figure 3), with an absolute increased risk of $22 \%$.

Finally, 203 matched pairs of morbidly obese versus normal weight patients were identified, and, again, all covariates were well balanced (Table 4). No difference was found in 30-day mortality between morbidly obese and normal weight patients (mean difference, $0.6 \% ; 95 \%$ CI, $-1.0 \%$ to $1.0 \%)$. This result was confirmed in the low-risk $(P=.4)$, medium-risk $(P=.9)$, and high-risk $(P=.8)$ patients. After a mean follow-up period of $6.0 \pm$ 4.2 years (range, $0-15$ ), morbidly obese patients tended to show an increased risk of late mortality compared with normal weight patients (HR, 1.36; 95\% CI, 0.74-2.49; $P=.3$; Figure 4), with an absolute increased risk of $36 \%$.

\section{DISCUSSION}

In contrast to previous reports suggesting the existence of an "obesity paradox" in patients undergoing $\mathrm{CABG},{ }^{4-7}$ the

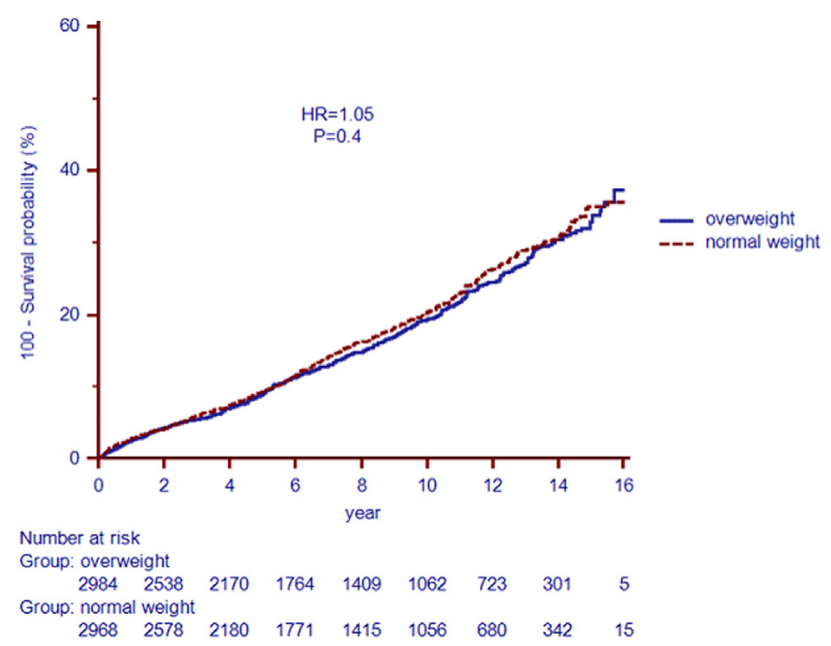

FIGURE 2. Kaplan-Meier curves with log-rank test result for late survival among matched normal weight and overweight subjects. $H R$, Hazard ratio. present propensity score-matched analysis showed that obesity did not confer any protective effect on early and late mortality when the baseline risk factors were well balanced. As previously reported, our data have shown that obesity and morbid obesity, per se, does not increase operative mortality even in medium- and high-risk patients, as identified by the EuroSCORE. However, obesity and morbid obesity were associated with a significantly increased risk of late mortality.

As shown in previous studies, the present analysis identified an inverse relationship between age and BMI categories, with obese patients younger than normal BMI patients. ${ }^{4-7}$ This finding was likely because of the influence of obesity-related metabolic alterations on accelerating the pathogenesis of coronary artery disease, resulting in a younger age at presentation.

Obesity is considered a major risk factor for cardiovascular disease, hypertension, and diabetes by National and International Committees. ${ }^{1}$ Thus, weight loss and prevention of obesity through dietary modifications and increased levels of exercise have been advocated. ${ }^{13,14}$ However, several studies of patients with established coronary artery disease, including patients undergoing $\mathrm{CABG}$, have shown more favorable short-term and long-term survival for patients with a higher BMI, implying a possible protective effect of obesity, defining the so-called obesity paradox. ${ }^{4,7}$ The main limitations of the previous reports were the small sample sizes and the significant differences in the baseline characteristics of patients belonging to different BMI categories.

Given the ever-increasing incidence of obesity and associated cardiometabolic disorders in Western countries, caution should be exercised in supporting a protective effect of obesity, unless conclusively proven true. By considering the demographic data of previously published studies, it could be argued that patients with a high BMI had better survival because of their better risk profile compared with that of 
TABLE 3. Baseline characteristics among normal weight and obese subjects after matching

\begin{tabular}{lcccc}
\hline & & & \multicolumn{2}{c}{ SMD } \\
\cline { 3 - 5 } Variable & Normal weight $(\mathbf{n}=\mathbf{1 6 0 9})$ & Obese $(\mathbf{n}=\mathbf{1 6 0 9})$ & Normal weight vs obese & Balance improvement $(\%)$ \\
\hline Age (y) & 67.3 & 67.7 & 0.04 & 87 \\
Female gender (\%) & 0.23 & 0.24 & 0.03 & 29 \\
Creatinine (mmol/L) & 105 & 104 & 0.01 & 25 \\
LV dysfunction (\%) & 0.47 & 0.46 & 0.03 & 32 \\
IDDM (\%) & 0.12 & 0.13 & 0.02 & 33 \\
COPD (\%) & 0.11 & 0.10 & 0.01 & 4.18 \\
Extracardiac arteriopathy (\%) & 0.12 & 0.10 & 0.07 & 90.21 \\
CVA (\%) & 0.01 & 0.01 & 0.05 & 181 \\
Nonelective surgery (\%) & 0.24 & 0.23 & 0.02 & 85 \\
Redo surgery (\%) & 0.036 & 0.037 & 0.005 & 78 \\
Year of operation & 2003.90 & 2003.63 & 0.05 & 73
\end{tabular}

$S M D$, Standardized mean difference; $L V$, left ventricle; $I D D M$, insulin-dependent diabetes mellitus; $C V A$, cerebrovascular accident; $C O P D$, chronic obstructive pulmonary disease.

normal weight patients, ${ }^{4-7}$ in particular, their younger age. Therefore, the so-called obesity paradox might simply be related to the fact that patients referred to surgery at a younger age have better survival in the short and medium term.

In observational studies comparing treatment options or the effect of various factors on clinical endpoints, the groups compared have often been different because of a lack of randomization. If the compared groups also differed between each other in respect to other variables that might have an effect on the chosen outcome measures, a direct comparison of the study groups would likely produce biased conclusions that might merely reflect the lack of initial comparability. To minimize the risk of such flawed conclusions, propensity score matching has been shown to produce estimates that are less biased, more robust, and more precise than the logistic regression estimates, especially for low rate outcomes such as death. ${ }^{15}$

In a relatively small sample size of heterogeneous patients, Stamou et $\mathrm{al}^{4}$ reported lower in-hospital mortality

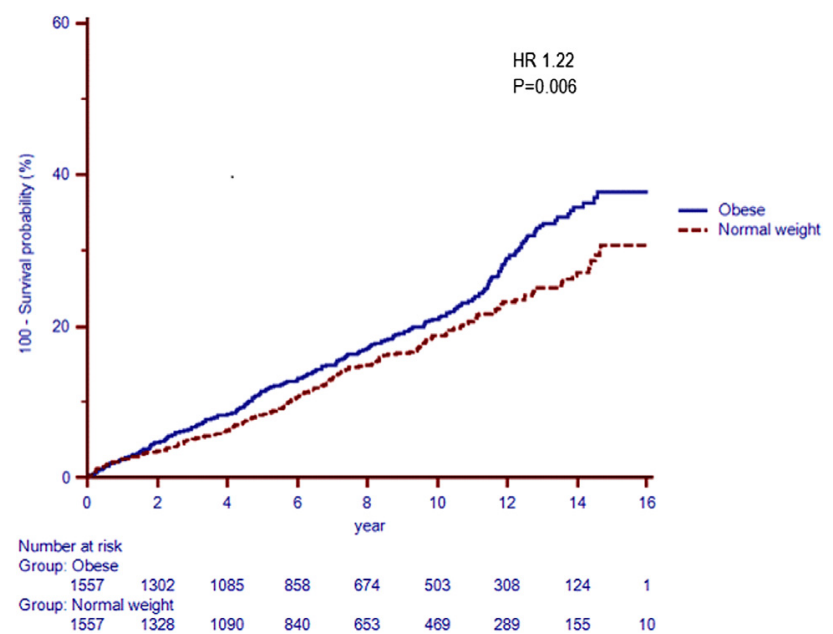

FIGURE 3. Kaplan-Meier curves with log-rank test result for late survival among matched normal weight and obese subjects. $H R$, Hazard ratio. and better long-term survival among overweight patients. Obese and morbidly obese patients had similar outcomes compared with normal weight patients. However, normal weight patients were significantly younger and had a higher incidence of congestive heart failure and left ventricular dysfunction. The investigators used propensity score matching for balancing covariates; however, the results of the balancing process were not reported. The better longterm survival among overweight patients was mainly related to the early phase, suggesting that residual differences in the patient risk profile could have influenced the results. Finally, even if no caliper of width was used for matching, it remains unclear why the matched sample size of normal weight patients was significantly smaller and thus affecting the power of the analysis. Sung et $\mathrm{al}^{7}$ concluded that overweight status is associated with improved survival after CABG. However, these investigators included a very small number of patients with significant differences in risk profiles between normal and overweight patients. The normal weight patients were significantly older and were more likely to have acute coronary syndrome, depressed left ventricular function, and decreased renal function. Furthermore, no propensity score matching was used to adjust for confounding factors. These limitations make their conclusion less reliable.

However, van Straten et $\mathrm{al}^{8}$ investigated the effect of the BMI on a large number of patients and concluded that overweight status did not confer a survival advantage. In contrast, they found that morbid obesity actually reduced late survival. ${ }^{8}$ Of note, the mean age among the normal weight and overweight patients was comparable. However, the results of the Bypass Angioplasty Revascularization Investigation trial $^{3}$ showed that although in-hospital complications were not adversely affected by obesity in the CABG population, the 5-year mortality progressively increased by up to fivefold with greater obesity.

The present propensity score analysis compared the effect of BMI in a large number of patients during 
TABLE 4. Baseline characteristics among normal weight and morbidly obese subjects after matching

\begin{tabular}{lcccc}
\hline & & & SMD \\
\cline { 3 - 5 } \multicolumn{1}{c}{ Variable } & Normal weight $(\mathbf{n}=\mathbf{2 0 3})$ & Morbidly obese $(\mathbf{n}=\mathbf{2 0 3})$ & $\begin{array}{c}\text { Normal weight vs } \\
\text { morbidly obese }\end{array}$ & Balance improvement $(\%)$ \\
\hline Age (y) & 61.0 & 61.0 & 0.001 & 99 \\
Female gender (\%) & 0.36 & 0.37 & 0.02 & 93 \\
Creatinine (mmol/L) & 101 & 100 & 0.02 & 88 \\
LV dysfunction (\%) & 0.49 & 0.50 & 0.03 & 71 \\
IDDM (\%) & 0.20 & 0.20 & 0.01 & 33 \\
COPD (\%) & 0.11 & 0.09 & 0.06 & 11 \\
Extracardiac arteriopathy (\%) & 0.09 & 0.07 & 0.05 & 49 \\
CVA (\%) & 0.004 & 0.009 & 0.05 & 89 \\
Nonelective surgery (\%) & 0.24 & 0.22 & 0.03 & 39 \\
Redo surgery (\%) & 0.029 & 0.049 & 0.09 & 73 \\
Year of operation (median) & 2003 & 2003 & 0.05 & \\
\hline
\end{tabular}

Data are presented as mean values, unless otherwise noted. SMD, Standardized mean difference; $L V$, left ventricle; $I D D M$, insulin-dependent diabetes mellitus; $C V A$, cerebrovascular accident; $C O P D$, chronic obstructive pulmonary disease.

long-term follow-up. In the present study, a high BMI was associated with a better risk profile according to the EuroSCORE, and this could be partially explained by the younger age of the obese patients. Before matching, overweight status appeared to be protective for early and late mortality. This result seemed to be primarily justified by a residual difference in the risk profile among the 2 groups, despite multivariate adjustment. In addition, when the BMI was considered as a continuous variable, the observed benefit on late survival was present for a short range of BMI values ( 27 and $28 \mathrm{~kg} / \mathrm{m}^{2}$ ), suggesting that confounding factors might have influenced this result.

After propensity matching, all baseline characteristics were well balanced among the BMI groups. In the matched sample, overweight status was no longer protective for early and late mortality. In accordance with

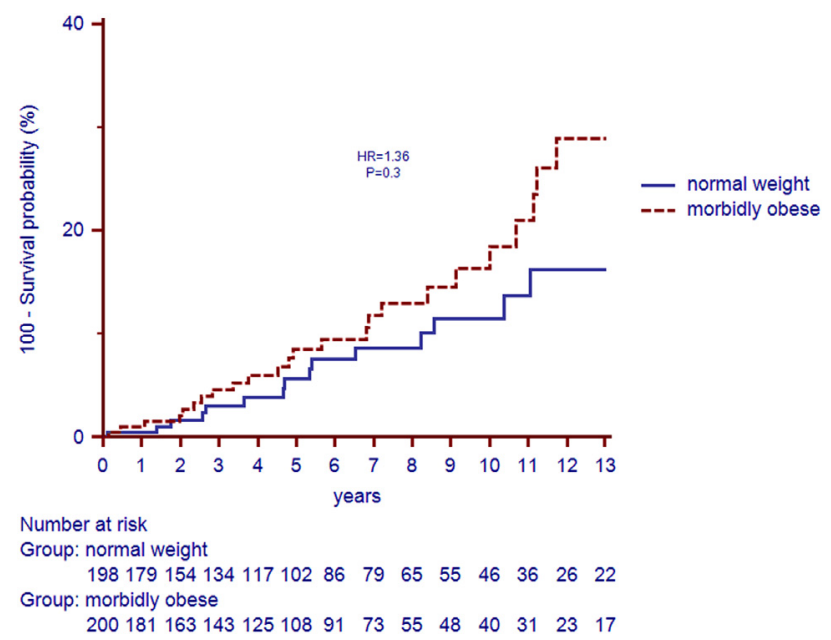

FIGURE 4. Kaplan-Meier curves with log-rank test result for late survival among matched normal weight and morbidly obese subjects. $H R$, Hazard ratio. previous reports, ${ }^{5,8}$ obesity and morbid obesity status per se did not increase perioperative mortality, regardless of the patient risk profile. However, both obesity and morbid obesity status were associated with an absolute increased risk of late death of $22 \%$ and $38 \%$, respectively.

\section{Study Limitations}

The present study has some limitations. Because of the small number of morbidly obese patients, the analysis of late mortality did not reach statistical significance. The rate of diabetic patients taking insulin was not significantly higher among patients with a high BMI. To explain this observation, it would be reasonable to speculate that obese patients with diabetes are treated more readily and aggressively with oral antidiabetic medications in an attempt to reduce insulin resistance.

We did not measure the changes in the BMI during the follow-up period; hence, no causality of the interrelationship between these parameters could be determined. In addition, obesity was defined only by the BMI in the present study, rather than by an actual measure of adiposity, such as the waist circumference. A recent study of the association of different measures of obesity and mortality reported that a high BMI is not directly associated with mortality and suggested that the waist circumference and waist-to-height ratio would be more reliable parameters to assess the mortality risk in patients with coronary artery disease. ${ }^{14}$ In addition, the combination of body fat and BMI was shown to be a better predictor of late survival than the BMI alone. ${ }^{16}$ However, the BMI is a widely available, simple, and practical obesity measurement, and numerous studies have used BMI as a surrogate measure of adiposity. However, an accurate quantitative measurement of lean and fat tissue and central adiposity should be performed in future studies. Finally, we were unable to assess the effect of 
cardiorespiratory fitness, which has been shown to influence the effect of obesity on late survival. ${ }^{17}$

\section{CONCLUSIONS}

Our results support the findings of previous studies showing that obesity is a major risk factor in cardiovascular disease, ${ }^{1,2}$ and did not show any protective effect of obesity toward early and late mortality in patients with cardiovascular disease, thus confuting the so-called obesity paradox. From the present findings, obese and morbidly obese patients should not be denied the benefits of CABG, because obesity per se does not increase early mortality, even in high-risk patients. However, robust postoperative weight loss programs should be strongly recommended for patients undergoing surgical myocardial revascularization, because obesity is associated with an absolute increased risk of $22 \%$ to $34 \%$ for late mortality after CABG.

The authors would like to thank Mr Malik Ahmed, Ms Sarah Powell, Ms Susan Davis and the entire Clinical Audit Department for their help in extracting the data. They would also like to acknowledge the contributions of all consultant cardiac surgeons operating at Papworth Hospital during the period of interest: S. Billing, P. Catarino, C. Choong, M. Codispoti, K. Dhital, J. Dunning, T. English, A. Goodwin, P. Jansz, D. Jenkins, S. Large, N. Moorjani, K. Nair, S. Nashef, C. Ng, A. Ritchie, B. Rosengard, C. Sudarshan, S. Tsui, J. Wallwork, and F. Wells.

\section{References}

1. Lavie CJ, Milani RV, Ventura HO. Obesity and cardiovascular disease: risk factor, paradox, and impact of weight loss. J Am Coll Cardiol. 2009;53:1925-32.

2. Taylor J. The obesity paradox. Eur Heart J. 2011;32:1575-6.

3. Gurm HS, Whitlow PL, Kip KE; BARI Investigators. The impact of body mass index on short- and long-term outcomes in patients undergoing coronary revascularization: insights from the Bypass Angioplasty Revascularization Investigation (BARI). J Am Coll Cardiol. 2002;39:834-40.
4. Stamou SC, Nussbaum M, Stiegel RM, Reames MK, Skipper ER, Robicsek F, et al. Effect of body mass index on outcomes after cardiac surgery: is there an obesity paradox? Ann Thorac Surg. 2011;91:42-7.

5. Le-Bert G, Santana O, Pineda AM, Zamora C, Lamas GA, Lamelas J. The obesity paradox in elderly obese patients undergoing coronary artery bypass surgery. Interact Cardiovasc Thorac Surg. 2011;13:124-7.

6. Gruberg L, Mercado N, Milo S, Boersma E, Disco C, van Es GA, et al. Arterial Revascularization Therapies Study Investigators. Impact of body mass index on the outcome of patients with multivessel disease randomized to either coronary artery bypass grafting or stenting in the ARTS trial: The obesity paradox II? Am J Cardiol. 2005;95:439-44.

7. Sung SH, Wu TC, Huang CH, Lin SJ, Chen JW. Prognostic impact of body mass index in patients undergoing coronary artery bypass surgery. Heart. 2011;97: 648-54.

8. van Straten AH, Bramer S, Soliman Hamad MA, van Zundert AA, Martens EJ, Schönberger JP, et al. Effect of body mass index on early and late mortality after coronary artery bypass grafting. Ann Thorac Surg. 2010;89:30-7.

9. Expert Panel on the Identification, Evaluation, and Treatment of Overweight in Adults. Clinical guidelines on the identification, evaluation, and treatment of overweight an obesity in adults. Am J Clin Nutr. 1998;68:899-917.

10. Roques F, Michel P, Goldstone AR, Nashef SA. The logistic EuroSCORE. Eur Heart J. 2003;24:881-2.

11. Ho D, Imai K, King G, Stuart E. Matching as nonparametric preprocessing for reducing model dependence in parametric causal inference. Polit Anal. 2007; 15:199-236.

12. Austin PC. A tutorial case study in propensity score analysis: an application to estimating the effect of in-hospital smoking cessation counseling on mortality. Multivariate Behav Res. 2011;46:119-51.

13. Wing RR, Lang W, Wadden TA, Safford M, Knowler WC, Bertoni AG, et al. Look AHEAD Research Group. Benefits of modest weight loss in improving cardiovascular risk factors in overweight and obese individuals with type 2 diabetes. Diabetes Care. 2011;34:1481-6.

14. Jago R, Mendoza JA, Chen T, Baranowski T. Longitudinal associations between BMI, waist circumference, and cardiometabolic risk in US youth: monitoring implications. Obesity (Silver Spring). 2013;21:E271-9.

15. Cepeda MS, Boston R, Farrar JT, Strom BL. Comparison of logistic regression versus propensity score when the number of events is low and there are multiple confounders. Am J Epidemiol. 2003;158:280-7.

16. Lavie CJ, De Schutter A, Patel D, Artham SM, Milani RV. Body composition and coronary heart disease mortality-an obesity or a lean paradox? Mayo Clin Proc. 2011;86:857-64.

17. McAuley PA, Kokkinos PF, Oliveira RB, Emerson BT, Myers JN. Obesity paradox and cardiorespiratory fitness in 12,417 male veterans aged 40 to 70 years. Mayo Clin Proc. 2010;85:115-21. 